BMJ Open Sport \& Exercise Medicine

\section{Effect of oral and transdermal oestrogen therapy on bone mineral density in functional hypothalamic amenorrhoea: a systematic review and meta-analysis}

To cite: Aalberg K, Stavem K, Norheim $\mathrm{F}$, et al. Effect of oral and transdermal oestrogen therapy on bone mineral density in functional hypothalamic amenorrhoea: a systematic review and meta-analysis. BMJ Open Sport \& Exercise Medicine 2021;7:e001112. doi:10.1136/ bmjsem-2021-001112

- Additional online supplemental material is published online only. To view, please visit the journal online (http://dx.doi.org/10.1136/ bmjsem-2021-001112).

Accepted 18 June 2021

Check for updates

(C) Author(s) (or their employer(s)) 2021. Re-use permitted under CC BY-NC. No commercial re-use. See rights and permissions. Published by BMJ.

For numbered affiliations see end of article.

Correspondence to Dr Karoline Aalberg; karoline_aalberg@hotmail.com

\section{ABSTRACT}

Background Female athletes might develop reduced bone mineral density (BMD) and amenorrhoea due to low energy intake.

Objective To systematically review the literature of randomised controlled trials (RCTs) assessing the effect of oestrogen oral contraceptives (OCP), conjugated oestrogens (CE) and transdermal estradiol (TE) on BMD in premenopausal women with functional hypothalamic amenorrhoea (FHA) due to weight loss, vigorous exercise and/or stress.

Methods A comprehensive literature search in PubMed, MEDLINE, Cochrane Library, Ovid and CINAHL from inception to 1 October 2020.

Data extraction and synthesis Two authors independently extracted data. When possible, the data were pooled in a random-effects meta-analysis.

Main outcomes Difference in BMD $\left(\mathrm{g} / \mathrm{cm}^{2}\right)$ at the lumbar spine.

Results Nine RCTs comprising 770 participants met the inclusion criteria; five studies applied OCP, two CE and two TE. Four RCTs (two OCP, two TE) found an increased BMD in premenopausal women with FHA, and five (three OCP, two $\mathrm{CE}$ ) found a decreased BMD compared with controls. A meta-analysis showed no difference in BMD between the treatment and control groups, (standardised mean difference (SMD) $0.30,95 \% \mathrm{Cl}-0.12$ to 0.73 ). A secondary analysis for change scores from baseline to first assessment point, showed a similar overall result (SMD $0.17,95 \% \mathrm{Cl}-0.16$ to 0.51 ). No serious adverse events were reported.

Conclusion The literature suggests that TE might increase lumbar BMD in premenopausal women with FHA, but pooled results revealed no effect of the intervention. The findings do not support oestrogen therapy to improve BMD in these patient groups.

\section{INTRODUCTION}

Exercise and physical activity are associated with positive effects on the human body, prevent diseases and improve general health. ${ }^{1}$ However, female athletes are at risk of developing the Female Athlete Triad, that is, low energy availability, menstrual dysfunction and

\section{Key messages}

What is already known

- Most commonly prescribed medication for bone mineral density in female athletes with functional hypothalamic amenorrhoea is oral contraceptive pills despite insufficient data on its effectiveness.

- Previous reviews have not investigated the effect of oestrogen therapy in the form of transdermal patch.

- Transdermal oestrogen does not undergo hepatic first-pass metabolism and is therefore not downregulating insulin-like growth factor-1 (as do oral contraceptives).

What are the new findings

- This study is the first to review the evidence of transdermal oestrogen patch on bone mineral density in premenopausal women with functional hypothalamic amenorrhoea.

- Oral contraceptives should not be used to improve bone health.

- Transdermal estradiol administration may be considered as second-line comanagement in order to optimise bone health in amenorrhoeic athletes.

reduced bone mineral density (BMD). ${ }^{2}$ The triad is a compound of the more complex syndrome Energy Deficiency in Sport (REDS), which refers to impaired physiological functioning as well as psychological consequences of relative energy deficiency. ${ }^{3}$

Many athletes reduce their energy intake intentionally in order to optimise body size and composition to achieve optimal performance. ${ }^{4}$ Prolonged energy deficiency, due to inadequate energy intake or excessive exercise, leads to hormonal disturbances of the hypothalamic-pituitary-gonadal (HPG) axis, which can result in functional hypothalamic amenorrhoea (FHA). ${ }^{5}$ FHA is a menstrual irregularity in absence of an organic cause, and occurs in $3 \%-66 \%$ of female athletes. ${ }^{6}$ Suppression of the HPG axis causes oestrogen deficiency, which increases rate of bone 
turnover, suppresses bone formation and prevents the attainment of peak bone mass in adolescents, ${ }^{78}$ contributing to increased fracture risk. ${ }^{19-11}$

The most effective strategy to normalise menstrual function and improve BMD in amenorrhoeic women is to restore energy balance and weight. ${ }^{3}{ }^{12}$ However, resumption of menses only gives minimal improvements in BMD. ${ }^{10}{ }^{11}$ FHA and hypo-oestrogenism during adolescence, a critical time of bone accrual, may lead to permanent deficits in peak bone mass. ${ }^{13}$ This underscores the need of alternative interventions for this population, including pharmacological management. The most commonly prescribed treatment for bone loss in premenopausal women with FHA is oral contraceptives (OCPs) or conjugated oestrogens (CE) to improve hypo-oestrogenic environment and, theoretically, protect bone. Systematic reviews have not found OCPs to significantly improve BMD in women with athletic FHA ${ }^{14-16}$ or anorexia nervosa (AN). ${ }^{141718}$ However, some recent randomised controlled trials (RCTs) have reported that transdermal estradiol (TE) is effective in increasing BMD in anorexic and amenorrhoeic premenopausal women. ${ }^{19} 20$

Therefore, we conducted a systematic review of clinical RCTs to determine the effect of oestrogen replacement, that is, OCP, CE and TE on BMD in premenopausal women with FHA.

\section{METHODS}

This systematic review identified oestrogen treatment strategies to improve BMD in women with FHA, and applied the preferred reporting items for systematic reviews and meta-analyses. ${ }^{21}$

\section{Search strategy and selection criteria}

A comprehensive literature search was conducted on PubMed, MEDLINE, Cochrane Library, Ovid and CINAHL including English or Scandinavian human RCTs prior to 1 October 2020. The key words functional hypothalamic amenorrhoea, BMD, osteoporosis, osteopaenia, oestrogen, hormone replacement therapy and OCP were used in various combinations. Two reviewers (KA and AC) independently reviewed the papers' titles, abstracts and full texts and resolved disagreements with a third reviewer $(\mathrm{FN})$.

Only RCTs with premenopausal women with functional hypothalamic oligo/amenorrhoea due to a negative energy balance, weight loss, vigorous exercise, eating disorders and/or stress who received oestrogen replacement therapy (OCP, CE or TE), were included.

Exclusion criteria were RCTs that enrolled patients with oligo/amenorrhoea resulting from anovulation, such as polycystic ovarian syndrome, diabetes, pituitary, renal and/or gastrointestinal disease, which can affect bone metabolism (BM). RCTs that enrolled patients on medications affecting BM such as glucocorticoids and anticonvulsants, were also excluded. We excluded RCTs that did not include controls or a placebo group, in addition to studies that did not include lumbar BMD as outcome measured by $\mathrm{g} / \mathrm{cm}^{2}$.

\section{Quality assessment and data extraction}

The methodological quality and internal validity of evidence were assessed individually by two reviewers (KA and AC) using the Physiotherapy Evidence Database (PEDro) scale, with a score range of $0-10 .^{22}$ An RCT with a score of $\geq 6$ was considered to be of high quality, while a score of $4-5$ was classified as fair quality, and $\leq 3$ as poor quality. The same two reviewers independently extracted data, with discrepancies resolved through consensus. Extracted information was tabulated and included country/year; study population, method, intervention, diagnostic test used, and main results.

\section{Outcomes}

The primary outcome was change in $\operatorname{BMD}\left(\mathrm{g} / \mathrm{cm}^{2}\right)$, measured using dual-energy X-ray absorptiometry (DXA) or dual photon absorptiometry (DPA) at the lumbar spine, because vertebral bodies have a high proportion of trabecular bone relative to cortical bone. ${ }^{23}$ Because trabecular bone has increased turnover compared with cortical bone, the effects of oestrogen may be apparent more rapidly in vertebral bodies than other anatomical sites such as the femoral shaft. ${ }^{24}$

\section{Data analyses}

The primary analysis identified a standardised mean difference (SMD) in each group receiving OCP, CE, $\mathrm{TE}$ versus control/placebo. We used data from the first assessment for each study, ranging from 9 to 12 months or 18-24 months after the baseline assessment. Random effects meta-analysis was conducted using the Hartung-Knapp-Sidak-Jonkman method, ${ }^{25}{ }^{26}$ which is recommended for analysis with few studies. ${ }^{27-29}$

Heterogeneity of the study results was analysed using the generalised $\mathrm{I}^{2}$ statistic; a percentage of $25 \%, 50 \%$ and $75 \%$ has been suggested to indicate low, medium, and high heterogeneity, respectively. ${ }^{30}$ We further examined heterogeneity using meta-regression with dichotomised independent variables. Here, assessed the impact of a priori identified sources of heterogeneity: (1) length of time from baseline to follow-up (18-24 months vs 9-12 months), (2) participants (competitive athlete/dancer vs anorexia/depression/anxiety), (3) age of participants (20-29 vs 15-19 years), (4) intervention in control arm (placebo control vs no intervention). We also prepared a funnel plot and used Egger's test for funnel plot asymmetry to identify possible publication bias with $\mathrm{p}<0.05$ suggesting asymmetry. ${ }^{31}$

As a secondary analysis, we analysed the SMD of change in BMD for the studies with available data using a similar method. In this analysis, we also created subgroups according to administration mode in the active study arm (patch vs pill).

\section{Patient and public involvement}

Patients were not involved in the development of the research question or its outcome measures, conduct of 
the research, or preparation of the manuscript. Findings will, however, be disseminated to patients via social media, relevant professional associations and news media.

\section{RESULTS}

\section{Study and subject selection and characteristics}

We identified 469 relevant abstracts and reviewed 76 of them in full text. Nine RCTs met our inclusion criteria (online supplemental figure S1). The included studies comprised 770 participants (age 11-35 years) with FHA; 448 had athletic amenorrhoea and $322 \mathrm{AN}$. The duration of the RCTs ranged from 9 to 24 months with a median of 12 months.

\section{Methodological quality}

The methodological PEDro score of the included RCTs ranged from 4 to 9 points out of a maximum of 10 points (mean 6.6 \pm 1.5 ) (table 1). Seven RCTs were considered of high quality ${ }^{19} 2032-36$ and two RCTs were considered of fair quality. ${ }^{37} 38$ Only one study concealed the outcome measures for the assessors, ${ }^{35}$ while four studies had $<15 \%$ drop-out. ${ }^{32-34} 37$

\section{BMD in pooled studies}

Online supplemental table S1 presents the data synthesis for study methodology and effectiveness for each of the nine RCTs.

When pooling the studies in the meta-analysis using the first assessment point, we found no difference in BMD between the treatment and control groups in the five studies included, that is, SMD of $0.30(95 \%$ CI -0.12 to 0.73 ), and only one study reported results for 18-24 months (online supplemental figure S2). The results for subgroups according to diagnostic classification, that is, anorexia/depression/anxiety vs competitive athletes/ dancers, was remarkably similar (online supplemental figure S3).

When exploring sources of heterogeneity in metaregression analysis, there was no difference in SMD between subgroups according to time of follow-up (18-24 months vs $9-12$ months) -0.74 ( $95 \%$ CI -1.97 to 0.49$)$, no intervention in controls vs placebo control -0.02 ( -0.95 to $0.90)$, age group included in the studies (20-29 vs 15-19 years) -0.34 (-1.18 to 0.49$)$, competitive athlete/dancer versus anorexia/depression/anxiety 0.00 (-0.94 to 0.94$)$. A funnel plot of the studies (online supplemental figure S5), and Egger's test $(\mathrm{p}=0.90)$, did not suggest asymmetry.

Four studies were included in the secondary analysis for change scores from baseline to first assessment point, with a pooled SMD of 0.17 (95\% CI -0.16 to 0.51 ). There was no apparent difference between subgroups according to time of follow-up (online supplemental figure S4), or mode of administration, i.e., patch vs pill (online supplemental figure $\mathrm{S} 6$ ).

Overall, the RCTs were fairly homogeneous, as indicated by an $\mathrm{I}^{2}<34 \%$ for subgroups in the pooled analyses (online supplemental figures S1-S4 and S6).

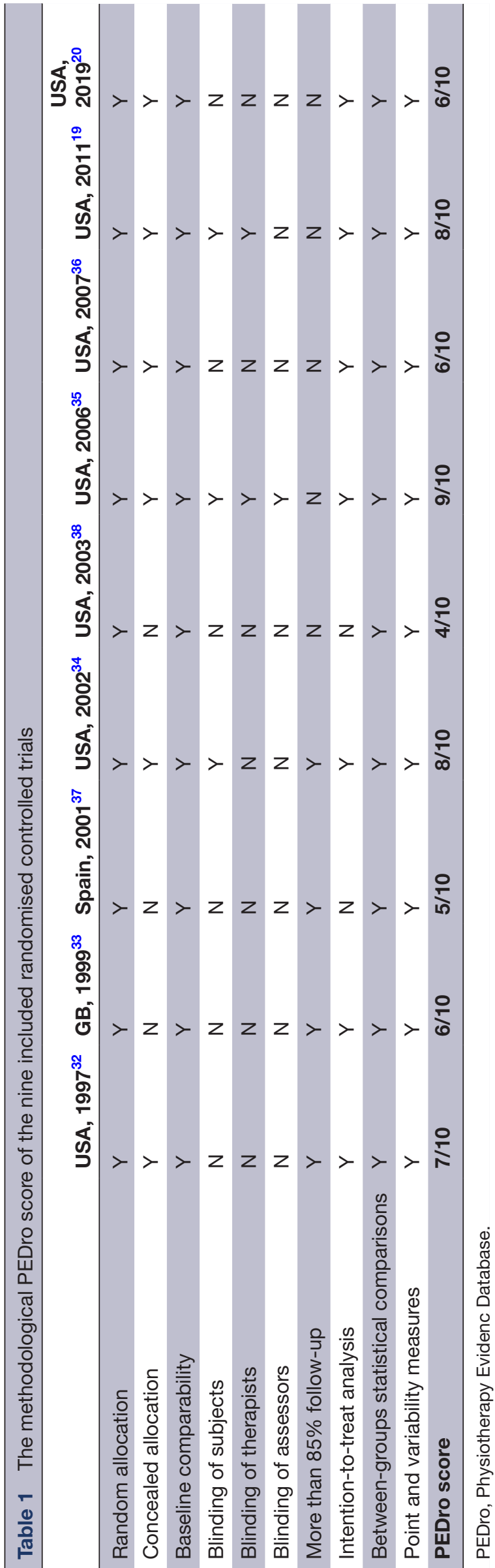


Oral contraceptives

Out of the five RCTs conducted with OCP, two studies showed a significant between-group change favouring the intervention group in lumbar BMD, ${ }^{32} 37$ and three studies showed no significant between-group effect (online supplemental table S1). ${ }^{34-36}$

\section{Conjugated oestrogens}

The two RCTs conducted with CE did not find a significant between-group difference in lumbar BMD in the intervention groups as compared with the control groups (online supplemental table S1). ${ }^{33}$ However, one study did find an increase in lumbar BMD of $5.7 \%$ in the $\mathrm{CE}$ group $(n=7)$ as compared with a $0.3 \%$ decrease in the control group $(n=9)$, with an adjusted calculated net effect of $1.5 \%$ after drop-outs and return of menses. ${ }^{33}$

\section{Transdermal estradiol}

Two high-quality RCTs applied TE patch (17- $\beta$ estradiol) and found a significant increased between-group change in spinal BMD in the active group in women with $\mathrm{AN}$ and FHA as compared with controls (online supplemental table S1). ${ }^{19} 20$

\section{Adverse events}

Six RCTs reported adverse events (AEs). ${ }^{32} 3738$ OCP and CE AEs were breast tenderness, headaches, bloating, nausea and mood swings. ${ }^{33} 3536$ TE AEs were headaches, bloating and erythema at patch application site. ${ }^{1920}$ None of the RCTs classified the severity or the duration of the AEs. No serious AEs were reported.

\section{DISCUSSION}

Our systematic review showed no significant increase in lumbar BMD by OCP and CE. However, the two TE patch RCTs showed an increase in spinal BMD in adolescents with athletic amenorrhoea and AN. ${ }^{1920}$ The meta-analysis found no pooled difference in BMD between the treatment and control groups.

\section{Methodological considerations}

All the included RCTs had overall small sample sizes, ranging from 24 to 150 participants with high drop-out rates (mean 22\% $\pm 17 \%$ ), which introduce attrition bias. Four RCTs did not report method of randomisation, ${ }^{20} 333738$ and three RCTs whether the allocation was concealed. ${ }^{33378}$ In one RCT participants were allocated to study arms by personal choice, which introduces selection bias. ${ }^{37}$

RCTs that include a placebo control group are advantageous to pragmatic studies and RCTs that use no interventions as control. To establish the true net effect of an intervention and quantify the actual placebo effect, one should ideally include both a placebo and a control group $^{39}{ }^{40}$; however, none of the included RCTs were designed this way. Five of the RCTs included a placebo arm. 1932343538 Reported reasons and justifications for not including a placebo control group were ethical considerations regarding contraceptive status and the high probability of un-blinding, as OCP may withdraw bleeding in amenorrhoeic subjects. ${ }^{34} 36$

The current systematic review included premenopausal women with FHA due to a negative energy balance caused by various underlying aetiologies, such as weight loss, vigorous exercise, eating disorders and/or stress. The various aetiologies makes a heterogeneous study population, but was decided on because patients with eating disorders and athletic amenorrhoea are believed to have similar aetiology, that is, chronic energy deficit where energy availability falls below a threshold of 20-30 $\mathrm{kcal} / \mathrm{kg}$ per day. ${ }^{41}$

The RCTs used different definitions of FHA, that is, no menstruation for $\geq 3$ months, ${ }^{19} 203435 \geq 5$ months ${ }^{38}$ and 6 months, ${ }^{32} 37$ while others defined FHA as $\leq 3$ cycles per year during the last $12^{36}$ or 18 months. ${ }^{33}$ This necessitates a concern about different baseline characteristics. The international classification of FHA defines it as the cessation of previously regular menstruation for 3 months or the cessation of irregular menses for 6 months. ${ }^{42}$ In comparison, the RCTs with anorexic subjects were diagnosed after the current Diagnostic and Statistical Manual of Mental Disorders, Fourth Edition criteria for classical $\mathrm{AN}$, which include no menstruation for $\geq 3$ months. ${ }^{43}$

Other factors affecting the results include differences in type, dose and formulation of hormones in the studies reviewed. The RCTs on OCP used various doses (range $0.03-35 \mathrm{mg}$ ) of the synthetic ethinyl estradiol, ${ }^{32}$ 34-37 in combination with different progestins, that is, norgestrel, ${ }^{36}$ norgestimate, ${ }^{35}$ norethindrone ${ }^{32} 34$ and desogestrel. ${ }^{37}$ The studies on CE used different doses of CE (estriol and estradiol) in combination with the progestins medroxyprogesterone acetate, ${ }^{38}$ and norethisterone. ${ }^{33}$ This makes it challenging to compare the results between studies, and it leaves uncertainty about the dose-response relationship. It is also difficult to determine whether the results with certainty were exclusive of oestrogens.

The current standard method for measuring BMD in the spine is DXA, ${ }^{44}$ but one of the included studies used DPA due to unavailability of a DXA instrument. ${ }^{38}$ DPA systems are less precise than DXA scanning and is reported to give higher measurements in BMD compared with DXA scans. ${ }^{45}$

A challenge pooling the results, were that some RCTs presented SE and not SD and had to be converted to a common unit.

Only two RCTs reported on fracture incidence, which is a clinically relevant outcome measure and gives clinicians information regarding absolute and relative risk ratio for patients with FHA. ${ }^{2036}$ However, due to the low incidence of fractures, generally the sample size in the RCTs were too small to encounter this side effect.

\section{Discussion of results}

Five RCTs assessed efficacy of OCP on lumbar BMD, ${ }^{32} 34-37$ of which two small studies demonstrated a mean positive effect in the intervention groups (total $n=51$ ) compared 
with control groups (total $\mathrm{n}=23$ ).$^{32} 37$ The mean lumbar BMD increased in these two studies by $3.9 \%$ in the OCP group with a net effect of $4.8 \%(\mathrm{SD} \pm 1.8)$. The net effect was higher because the control group had decreases in BMD compared with baseline. However, in contrast to all the other RCTs included in the study, none of these studies reported on resumption of normal menstruation during the 12-month follow-up. This introduces a large bias, as resumption of menses is a clinical sign of recovery from energy deficiency, which may increase vertebral BMD of approximately $6.5 \%$ over 15 months. ${ }^{46}$ Previous systematic reviews and meta-analysis have similarly found OCP to be ineffective in increasing BMD in patient with $\mathrm{AN}^{1718}$ or FHA. ${ }^{1416}$

Both RCTs on TE patch with cyclic progesterone were well conducted and showed a significant improvement in BMD in amenorrhoeic girls at 12 and 18-month follow-up with a mean within-group change in the treatment groups of $2.51 \%(\mathrm{n}=98)$ and a mean net effect of $2.50 \%$ (SD $\pm 0.28) .{ }^{19}{ }^{20}$ One of the TE patch RCTs did also include a placebo patch group, which is a better approach for assessing efficacy and safety data. ${ }^{19}$

\section{Treatment strategies and clinical implications}

Although TE patch with cyclic progesterone show promising results, there is still uncertainty regarding the efficacy. Weight and energy balance restoration is the first-line treatment of FHA, and early detection is critical in order to prevent life-long ramifications on bone health. ${ }^{47}$ Relative risk for stress fractures and osteoporotic fractures of the hip and spine is two to four times higher in amenorrhoeic than eumenorrheic athletes. ${ }^{48}$ Two studies suggested that increasing energy intake to more than $30 \mathrm{kcal} / \mathrm{kg}$ of fat free mass per day may restore the menstrual cycle. ${ }^{41} 49$

The International Olympic Committee statement on RED-S from 2018 recommends non-pharmacological management in terms of a multidisciplinary approach is recommended as first-line management for recovery. ${ }^{3} \mathrm{~A}$ multidisciplinary team should include a primary healthcare practitioner, sport physiologist, a sport dietitian and mental health practitioner. ${ }^{50}$ If menstrual cycles is not restored after 1 year of specific individualised modification through a multidisciplinary team approach, second-line pharmacological interventions can be supplemented to prevent further bone loss. ${ }^{23}$

In cases where second-line management is warranted, clinicians need to consider different metabolic factors. First, oral oestrogens undergo hepatic first-pass metabolism and downregulate insulin-like growth factor 1 (IGF-1), which is a bone-trophic hormone that increases during puberty and is critical in order to enhance bone accrual. ${ }^{51}$ Second, athletes with FHA already have diminished levels of IGF- $1,{ }^{52}$ and a further reduction in IGF-1 levels by OCP is likely to limit the otherwise antiresorptive effects of oestrogen. ${ }^{53}$ Finally, ethinyl estradiol has a dose-dependent stimulatory effect on hepatic sex hormone-binding globulin production, which may lower physiologic and bioavailable estradiol in the female. ${ }^{54}$ In comparison, TE do not undergo hepatic first-pass metabolism and is therefore not downregulating IGF- $1 .^{55}{ }^{56} \mathrm{In}$ fact, studies have found that TE may even increase levels of circulating IGF- $1 .^{57}$

Thus, based on our results, TE therapy with cyclic oral progestin can be considered in a short period as secondline supplement for young adolescents in cases where first-line management fall short.

\section{Limitations}

The present study might have possible biases. Although we did perform a comprehensive and systematic search, we may have missed RCTs published in non-English languages. Furthermore, the body of evidence is small, since only a total of 770 subjects are included with a median follow-up of 12 months. We included subjects with various conditions that were classified as AN (clinical eating disorder) and athletic amenorrhoea (subclinical eating disorder). Nevertheless, the underlying physiology is considered to be similar, that is, energy deficit. Thus, the current systematic review is based on an inhomogeneous patient population, and results should be interpreted with caution.

\section{CONCLUSION}

Current evidence does not support oral oestrogen replacement in the form of OCP or CE to improve BMD in patients with FHA. Pharmacological treatment in the form of TE patch can be considered as second-line comanagement in order to optimise bone health, but only if weight and energy balance restoration through a non-pharmacological multidisciplinary management approach fall short within 1 year. Further longitudinal and prospective studies are needed to confirm the effect of TE therapy on BMD.

\section{Author affiliations \\ ${ }^{1}$ Atlasklinikken, Oslo, Norway \\ ${ }^{2}$ Institute of Clinical Medicine, Akershus University Hospital, Lorenskog, Norway ${ }^{3}$ Department of Pulmonary Medicine, University of Oslo, Akershus University Hospital, Lorenskog, Norway \\ ${ }^{4}$ Department of Nutrition, University of Oslo, Institute of Basic Medical Sciences, Oslo, Norway \\ ${ }^{5} \mathrm{Head}$ and Neck Research Group, Division for Research and Innovation, Akershus University Hospital, Lorenskog, Norway \\ ${ }^{6}$ Department for Interdisciplinary Health Sciences, Institute of Health and Society, University of Oslo Faculty of Medicine, Oslo, Norway}

Acknowledgements We would like to acknowledge Halvor Sørbye, Maja Eidsmo Bjørnli, Patrick Johansen Hammeren, Anja Johansen, Knut Andersen, Hilde Hamre, Joakim Hempel Hansen, Hege Herstad, Stein Tore Krey, Celina Maria Rangela Lothe, Vilhelm Scheel, Ole Sommerset, Andreas Welander, Per-Øyvind Granlund Marius Riiber Eikeland, Lars Havig Berge, Tale Haugse, Elisabeth Loeng Hayfield, Lovisa Marie Klingenberg, Elisabeth Ohlgren Tidemann, Daniel Vestøl and Head and Neck Research Group for their generous donation to allow us to submit the paper to an open access journal.

Contributors KA conceived the review and drafted the initial manuscript. KA and AC performed the methodological assessment of the included studies. KA, MBR and $\mathrm{AC}$ synthesised the data in table. KS performed the statistical analysis. All authors have revised the paper for critical content and approved the final version. 
Funding The authors have not declared a specific grant for this research from any funding agency in the public, commercial or not-for-profit sectors.

Competing interests None declared.

Patient consent for publication Not required.

Provenance and peer review Not commissioned; externally peer reviewed.

Open access This is an open access article distributed in accordance with the Creative Commons Attribution Non Commercial (CC BY-NC 4.0) license, which permits others to distribute, remix, adapt, build upon this work non-commercially, and license their derivative works on different terms, provided the original work is properly cited, appropriate credit is given, any changes made indicated, and the use is non-commercial. See: http://creativecommons.org/licenses/by-nc/4.0/.

\section{ORCID iDs}

Karoline Aalberg http://orcid.org/0000-0002-6433-999X

Aleksander Chaibi http://orcid.org/0000-0003-2566-5984

\section{REFERENCES}

1 Nichols DL, Bonnick SL, Sanborn CF. Bone health and osteoporosis. Clin Sports Med 2000;19:233-49.

2 De Souza MJ, Nattiv A, Joy E, et al. 2014 female athlete triad coalition consensus statement on treatment and return to play of the female athlete triad: 1st International Conference held in San Francisco, California, may 2012 and 2nd International Conference held in Indianapolis, Indiana, may 2013. Br J Sports Med 2014:48:289

3 Mountjoy M, Sundgot-Borgen JK, Burke LM, et al. IOC consensus statement on relative energy deficiency in sport (RED-S): 2018 update. Br J Sports Med 2018:52:687-97.

4 Sundgot-Borgen J. Risk and trigger factors for the development of eating disorders in female elite athletes. Med Sci Sports Exerc 1994;26:414???419-9.

5 Meczekalski B, Podfigurna-Stopa A, Warenik-Szymankiewicz $\mathrm{A}$, et al. Functional hypothalamic amenorrhea: current view on neuroendocrine aberrations. Gynecol Endocrinol 2008;24:4-11.

6 Yeager KK, Agostini R, Nattiv A, et al. The female athlete triad: disordered eating, amenorrhea, osteoporosis. Med Sci Sports Exerc 1993;25:775-7.

7 Bailey DA, McKay HA, Mirwald RL, et al. A six-year longitudinal study of the relationship of physical activity to bone mineral accrual in growing children: the University of Saskatchewan bone mineral accrual study. J Bone Miner Res 1999;14:1672-9.

8 Baxter-Jones ADG, Faulkner RA, Forwood MR, et al. Bone mineral accrual from 8 to 30 years of age: an estimation of peak bone mass. J Bone Miner Res 2011;26:1729-39.

9 Biller BM, Baum HB, Rosenthal DI, et al. Progressive trabecular osteopenia in women with hyperprolactinemic amenorrhea. J Clin Endocrinol Metab 1992;75:692-7.

10 Miller KK, Klibanski A. Amenorrheic bone loss. J Clin Endocrinol Metab 1999;84:1775-83.

11 Roupas ND, Georgopoulos NA. Menstrual function in sports. Hormones 2011;10:104-16.

12 Sundgot-Borgen J, Torstveit MK. The female football player, disordered eating, menstrual function and bone health. Br J Sports Med 2007;41 Suppl 1:i68-72

13 Misra M, Klibanski A. Anorexia nervosa and bone. J Endocrinol 2014;221:R163-76.

14 Liu SL, Lebrun CM. Effect of oral contraceptives and hormone replacement therapy on bone mineral density in premenopausal and perimenopausal women: a systematic review. Br J Sports Med 2006:40:11-24.

15 Vescovi JD, Jamal SA, De Souza MJ. Strategies to reverse bone loss in women with functional hypothalamic amenorrhea: a systematic review of the literature. Osteoporos Int 2008;19:465-78.

16 Altayar O, Al Nofal A, Carranza Leon BG, et al. Treatments to prevent bone loss in functional hypothalamic amenorrhea: a systematic review and meta-analysis. J Endocr Soc 2017;1:500-11.

17 Sim LA, McGovern L, Elamin MB, et al. Effect on bone health of estrogen preparations in premenopausal women with anorexia nervosa: a systematic review and meta-analyses. Int $J$ Eat Disord 2010;43:218-25.

18 Robinson L, Aldridge V, Clark EM, et al. Pharmacological treatment options for low bone mineral density and secondary osteoporosis in anorexia nervosa: a systematic review of the literature. J Psychosom Res 2017;98:87-97.

19 Misra M, Katzman D, Miller KK, et al. Physiologic estrogen replacement increases bone density in adolescent girls with anorexia nervosa. J Bone Miner Res 2011;26:2430-8.
20 Ackerman KE, Singhal V, Baskaran C, et al. Oestrogen replacement improves bone mineral density in oligo-amenorrhoeic athletes: a randomised clinical trial. Br J Sports Med 2019;53:229-36.

21 Liberati Aet al. The PRISMA statement for reporting systematic reviews and meta-analyses of studies that evaluate health care interventions: explanation and elaboration. Ann Intern Med 2009;151:W-65-W-94.

22 Moseley AM, Herbert RD, Sherrington C, et al. Evidence for physiotherapy practice: a survey of the physiotherapy evidence database (PEDro). Australian Journal of Physiotherapy 2002;48:43-9.

23 Nakano T, Tabata Y, Umakoshi Y. Texture and Bone Reinforcement. In: Encyclopedia of materials: science and technology. Elsevier, Oxford, 2005.

24 Israel O, Lubushitzky R, Frenkel A, et al. Bone turnover in cortical and trabecular bone in normal women and in women with osteoporosis. J Nucl Med 1994;35:1155-8.

25 Hartung J. An alternative method for meta-analysis. Biometrical Journal 1999;41:901-16

26 Knapp G, Hartung J. Improved tests for a random effects metaregression with a single covariate. Stat Med 2003;22:2693-710.

27 IntHout J, loannidis JPA, Borm GF. The Hartung-KnappSidik-Jonkman method for random effects meta-analysis is straightforward and considerably outperforms the standard DerSimonian-Laird method. BMC Med Res Methodol 2014;14:25

28 Bender R, Friede T, Koch A, et al. Methods for evidence synthesis in the case of very few studies. Res Synth Methods 2018;9:382-92.

29 Veroniki AA, Jackson D, Bender R, et al. Methods to calculate uncertainty in the estimated overall effect size from a random-effects meta-analysis. Res Synth Methods 2019;10:23-43.

30 Higgins JPT, Thompson SG, Deeks JJ, et al. Measuring inconsistency in meta-analyses. BMJ 2003;327:557-60.

31 Egger M, Smith GD, Phillips AN. Meta-Analysis: principles and procedures. BMJ 1997;315:1533-7.

32 Hergenroeder AC, Smith EO, Shypailo R, et al. Bone mineral changes in young women with hypothalamic amenorrhea treated with oral contraceptives, medroxyprogesterone, or placebo over 12 months. Am J Obstet Gynecol 1997;176:1017-25.

33 Gibson JH, Mitchell A, Reeve J, et al. Treatment of reduced bone mineral density in athletic amenorrhea: a pilot study. Osteoporos Int 1999;10:284-9.

34 Grinspoon S, Thomas L, Miller K, et al. Effects of recombinant human IGF-I and oral contraceptive administration on bone density in anorexia nervosa. J Clin Endocrinol Metab 2002;87:2883-91.

35 Strokosch GR, Friedman AJ, Wu S-C, et al. Effects of an oral contraceptive (norgestimate/ethinyl estradiol) on bone minera density in adolescent females with anorexia nervosa: a double-blind placebo-controlled study. J Adolesc Health 2006;39:819-27.

36 Cobb KL, Bachrach LK, Sowers M, et al. The effect of oral contraceptives on bone mass and stress fractures in female runners. Med Sci Sports Exerc 2007;39:1464-73.

37 Castelo-Branco C, Vicente JJ, Pons F, et al. Bone mineral density in young, hypothalamic oligoamenorrheic women treated with oral contraceptives. J Reprod Med 2001;46:875-9.

38 Warren MP, Brooks-Gunn J, Fox RP, et al. Persistent osteopenia in ballet dancers with amenorrhea and delayed menarche despite hormone therapy: a longitudinal study. Fertil Steril 2003;80:398-404.

39 Chaibi A, Russell MB. Manual therapies for cervicogenic headache: a systematic review. J Headache Pain 2012;13:351-9.

40 Chaibi A, Tuchin PJ, Russell MB. Manual therapies for migraine: a systematic review. J Headache Pain 2011:12:127-33.

41 Loucks AB, Thuma JR. Luteinizing hormone Pulsatility is disrupted at a threshold of energy availability in regularly menstruating women. $J$ Clin Endocrinol Metab 2003;88:297-311.

42 Klein DA, Paradise SL, Reeder RM. Amenorrhea: a systematic approach to diagnosis and management. Am Fam Physician 2019;100:39-48.

43 Substance Abuse and Mental Health Services Administration. In: Disturbance D-ClfCSE, ed. US: Rockville (MD): Substance Abuse and Mental Health Services Administration (US), 2016:Table 19, DSM-IV to DSM-5 Anorexia Nervosa Comparison.

44 Sheu A, Diamond T. Bone mineral density: testing for osteoporosis. Aust Prescr 2016;39:35-9.

45 Wahner HW, Dunn WL, Brown ML, et al. Comparison of dualenergy X-ray absorptiometry and dual photon absorptiometry for bone mineral measurements of the lumbar spine. Mayo Clin Proc 1988;63:1075-84.

46 Drinkwater BL, Nilson $\mathrm{K}$, Ott S, et al. Bone mineral density after resumption of menses in amenorrheic athletes. JAMA 1986:256:380-2. 
47 Gordon CM, Ackerman KE, Berga SL, et al. Functional hypothalamic amenorrhea: an endocrine Society clinical practice guideline. J Clin Endocrinol Metab 2017;102:1413-39.

48 Warren MP, Brooks-Gunn J, Fox RP, et al. Osteopenia in exerciseassociated amenorrhea using ballet dancers as a model: a longitudinal study. J Clin Endocrinol Metab 2002;87:3162-8.

49 Dueck CA, Matt KS, Manore MM, et al. Treatment of athletic amenorrhea with a diet and training intervention program. Int $J$ Sport Nutr 1996;6:24-40.

50 Joy E, De Souza MJ, Nattiv A, et al. 2014 female athlete triad coalition consensus statement on treatment and return to play of the female athlete triad. Curr Sports Med Rep 2014;13:219-32.

51 Leung K-C, Johannsson G, Leong GM, et al. Estrogen regulation of growth hormone action. Endocr Rev 2004;25:693-721.

52 Misra M, Miller KK, Almazan C, et al. Alterations in cortisol secretory dynamics in adolescent girls with anorexia nervosa and effects on bone metabolism. J Clin Endocrinol Metab 2004;89:4972-80.
53 Lebow J, Sim L. The influence of estrogen therapies on bone mineral density in premenopausal women with anorexia nervosa and amenorrhea. Vitam Horm 2013;92:243-57.

54 van der Vange N, Blankenstein MA, Kloosterboer HJ, et al. Effects of seven low-dose combined oral contraceptives on sex hormone binding globulin, corticosteroid binding globulin, total and free testosterone. Contraception 1990;41:345-52.

55 Weissberger AJ, Ho KK, Lazarus L. Contrasting effects of oral and transdermal routes of estrogen replacement therapy on 24-hour growth hormone $(\mathrm{GH})$ secretion, insulin-like growth factor I, and $\mathrm{GH}$ binding protein in postmenopausal women. J Clin Endocrinol Metab 1991;72:374-81.

56 Cardim HJ, Lopes CM, Giannella-Neto D, et al. The insulin-like growth factor-I system and hormone replacement therapy. Fertil Steril 2001;75:282-7.

57 Isotton AL, Wender MCO, Casagrande A, et al. Effects of oral and transdermal estrogen on IGF1, IGFBP3, IGFBP1, serum lipids, and glucose in patients with hypopituitarism during $\mathrm{GH}$ treatment: a randomized study. Eur J Endocrinol 2012;166:207-13. 